University of Massachusetts Amherst

ScholarWorks@UMass Amherst

Chemical Engineering Faculty Publication

Series

2020

\title{
Incorporation of proteins into complex coacervates
}

Whitney C. Blocher McTigue

University of Massachusetts Amherst

Sarah L. Perry

University of Massachusetts Amherst

Follow this and additional works at: https://scholarworks.umass.edu/che_faculty_pubs

\section{Recommended Citation}

Blocher McTigue, Whitney C. and Perry, Sarah L., "Incorporation of proteins into complex coacervates" (2020). Methods in Enzymology. 890.

https://doi.org/10.1016/bs.mie.2020.06.006

This Article is brought to you for free and open access by the Chemical Engineering at ScholarWorks@UMass Amherst. It has been accepted for inclusion in Chemical Engineering Faculty Publication Series by an authorized administrator of ScholarWorks@UMass Amherst. For more information, please contact scholarworks@library.umass.edu. 


\title{
Incorporation of Proteins into Complex Coacervates
}

\author{
Whitney C. Blocher McTigue and Sarah L. Perry \\ wblocher@umass.edu and perrys@engin.umass.edu
}

\begin{abstract}
:
Complex coacervates have found a renewed interest in the past few decades in various fields such as food and personal care products, membraneless cellular compartments, the origin of life, and, most notably, as a mode of transport and stabilization of drugs. Here, we describe general methods for characterizing the phase behavior of complex coacervates and quantifying the incorporation of proteins into these phase separated materials.
\end{abstract}




\section{Table of Contents}

Incorporation of Proteins into Complex Coacervates ................................................................. 1

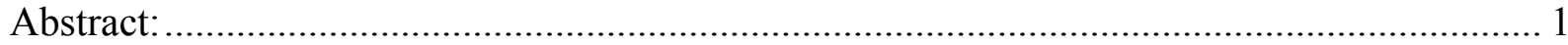

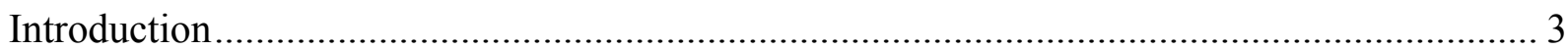

Complex Coacervation and Protein Incorporation ........................................................... 3

Complex Coacervate Phase Behavior .............................................................................. 3

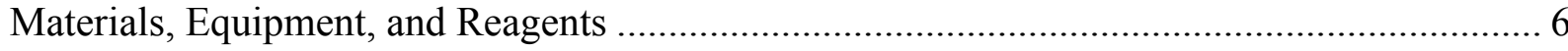

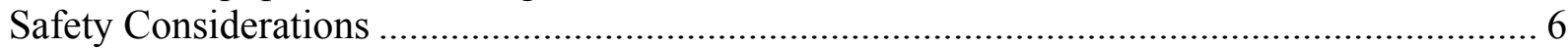

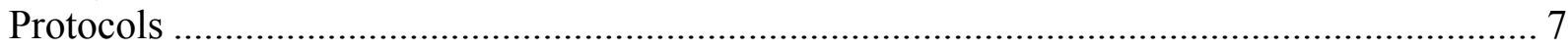

Characterizing Coacervate Phase Behavior ..................................................................... 7

Polymer-Polymer Stoichiometry Experiments ……………........................................ 7

Polymer-Polymer Salt Resistance Experiments.............................................................. 10

Experimental Protocol for Coacervate Samples (no protein) ....................................... 11

Polymer-Polymer-Protein Stoichiometry Experiments ................................................... 12

Polymer-Polymer-Protein Salt Resistance Experiments................................................... 13

Experimental Protocol for Coacervate Samples (with protein) .................................... 13

Quantifying Protein Incorporation into Complex Coacervates ........................................... 14

Bradford Assay ............................................................................................... 14

Experimental Protocol for Protein Quantification Using the Bradford Assay ............. 16

Utilizing Absorbance at $280 \mathrm{~nm}$........................................................................... 17

Experimental Protocol for Protein Quantification Using A280 ................................... 18

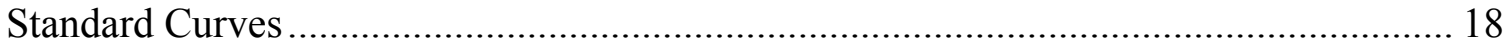

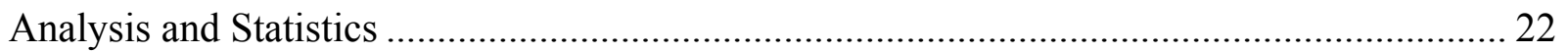

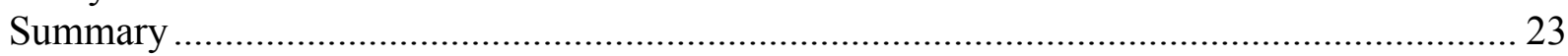

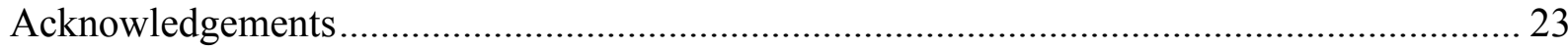

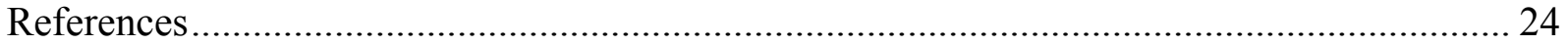




\section{Introduction}

The encapsulation of proteins and other biomacromolecules is an area of tremendous activity, as such materials are finding increasing utility in applications such as drug delivery, environmental remediation, personal care products and biocatalysis. Proteins are generally very sensitive to their environment, and typical methods used for encapsulation can decrease or even destroy the activity of these molecules. Complex coacervation is a method that can be used to encapsulate proteins without using harsh conditions that may denature the protein cargo. This method of sequestration is a viable platform for a variety of different areas such as food science,,${ }^{1,2}$ personal care products, ${ }^{3,4}$ and medicine, ${ }^{5}$ because of the ability to generate biocompatible formulations and drive high levels of encapsulation without the need of organic solvents. ${ }^{6-9}$

\section{Complex Coacervation and Protein Incorporation}

Complexation occurs when oppositely charged polyelectrolytes interact under favorable conditions such that the electrostatic attraction and entropic gains can drive phase separation. Coacervation is a purely aqueous strategy that can also be leveraged for the triggerable release protein cargo. ${ }^{10-12}$ These materials are versatile and have been shown usable for various delivery techniques such as an injectable protein carrier ${ }^{13-15}$ and for oral delivery. ${ }^{16,17}$ This method will focus on using complex coacervation as an aqueous protein encapsulation technique, and experimental strategies related to characterizing the concentration of protein present in such formulations.

\section{Complex Coacervate Phase Behavior}

There are many variables that alter the ability of polyelectrolytes to undergo complex coacervation, including the salt concentration, $\mathrm{pH}$, charge density and chemistry of the polymers, etc. Here, we will describe an experimental strategy for characterizing aspects of the phase behavior of complex coacervates, without consideration for other formulation-relevant questions such as the size and temporal stability of a dispersion of coacervate droplets etc. One consequence of this experimental focus is the use of concentration on an ionizable monomer basis, rather than units of mass of polymer per volume that are more typical when considering polymeric materials. Furthermore, this discussion assumes that the coacervate materials in question are able to fully equilibrate (i.e., form liquid droplets, rather than kinetically-trapped gels or solid complexes).

Complex coacervation involves the interaction of oppositely-charged polyelectrolytes. Thus, while physical and chemical aspects of the polymer such as length, charge density and/or degree of ionization, as well as hydrophobicity can modulate the phase behavior, we can typically rely on general intuition regarding electrostatic effects and charge neutrality. For complex coacervation, the ability to phase separate will be maximized when an equal number of oppositely-charged groups are present. While this condition may be straightforward to predict with some simple polymer systems, factors such as $\mathrm{pH}$-dependent degree of ionization can play a role. These effects are highlighted in Figurela,b, which show schematic depictions of a two dimensional phase diagram as a function of the concentration of polycation and polyanion, with the concentration of salt present in the system defining the contours. The height (i.e., salt stability) of the two-phase region is maximized along the line of equimolar charge (black line). For systems where all of the potentially ionizable monomers are charged, this results in a symmetric phase diagram (Figure 1a). However, if the $\mathrm{pH}$ of the solution has been changed such that only half of the ionizable groups on one of the polymers are charged (e.g., the apparent pKa 
of the polycation), then the phase diagram becomes asymmetric, as in Figure $1 \mathrm{~b}$.

(a)

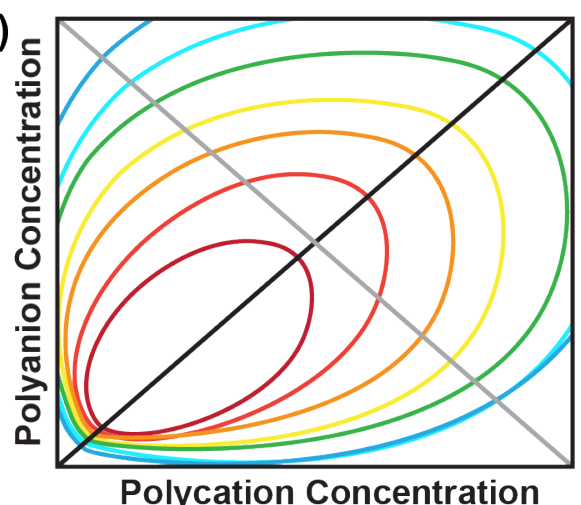

(c)

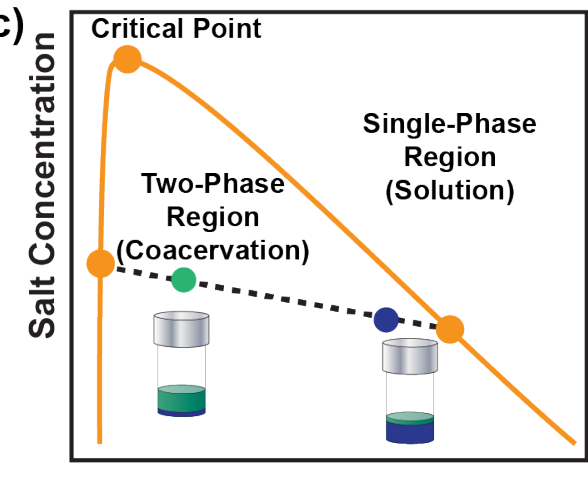

Polymer Concentration (b)

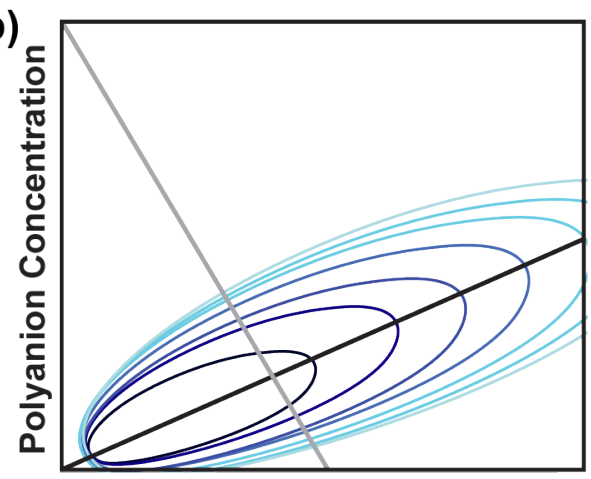

Polycation Concentration

(d)

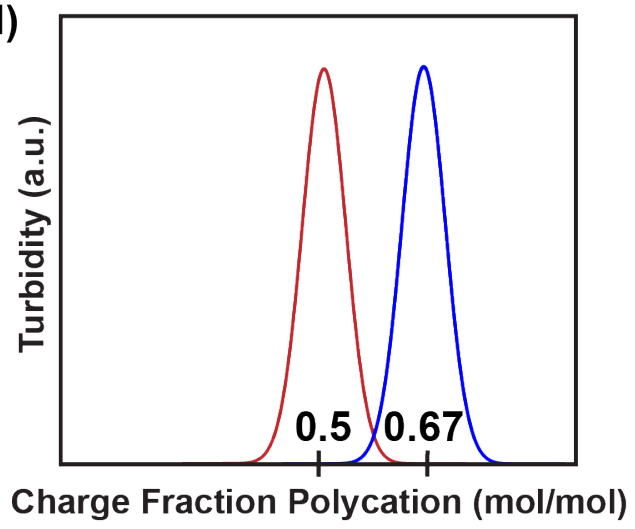

Figure 1: A schematic contour plot of the three-dimensional phase envelope for complex coacervation as a function of relative polycation concentration, polyanion concentration, and salt concentration (shown as contours) for (a) a system where the relative degree of ionization of the polycation and polyanion is the same, and (b) a system where the relative degree of ionization for the polycation is half that of the polyanion. The black line in both plots indicates the salt-polymer phase behavior for a mixture of equal numbers of ionized cationic and anionic species, shown as the binodal curve in (c). The grey line traces out the effect of changing the charge stoichiometry of the system. (c) A schematic illustration of a typical salt versus polymer concentration phase diagram defined by the black lines in $(\mathbf{a}, \mathbf{b})$. Coacervation occurs in the two-phase region beneath the binodal curve. A sample prepared within this two-phase region will phase separate into a polymer-dense coacervate phase and a polymer-poor supernatant phase, connected by a tie-line. While identical coacervate and supernatant phases will be formed from any sample prepared along such a tie-line, the relative position on the line dictates the fraction of the resulting sample that will be coacervate vs. supernatant, as per the lever rule. A sample prepared at relatively high polymer concentration (blue dot) will produce a much larger volume of coacervate than one prepared at lower polymer concentrations (green). The relative volumes of coacervate (blue) and supernatant (green) are indicated in the depicted vials. (d) Schematic depiction of the results of a stoichiometry experiment, which tests the effect of changing the relative amounts of polycation and polyanion at constant total polymer concentration and constant solution conditions. The turbidity signal maps out conditions where phase separation occurs. For the system where the degree of ionization of the polymeric species is the same, a maximum in turbidity is observed at a charge fraction of 0.5 (red, corresponding to the grey line in (a). This result shifts to a cationic charge fraction of 0.67 if the degree of ionization of the polycation is half that of the polyanion (blue, corresponding to the grey line in (b)).

These same trends can be observed through a "stoichiometry" experiment, which typically uses turbidity to determine the polymer ratio that gives maximum coacervate yield. ${ }^{18-24} \mathrm{~A}$ stoichiometry experiment varies the relative amount of polycation to polyanion while keeping the total polymer concentration constant (corresponding to the grey lines in Figure 1a,b). For systems where the polycation and polyanion can be considered as fully ionized (or have equal levels of ionization), the peak in the turbidity would be expected at a mole fraction of 0.5 with respect to one of the polymer species, or a 1:1 equimolar ratio (Figure 1d, red curve). However, if one of the polymer species is only half charged, this will result in a shift in the observed signal. 
For instance, in the example shown in Figure $1 \mathrm{~b}$ where only half of the monomers on the polycation are ionized, a turbidity peak would be observed at a charge fraction of 0.67 , corresponding to the condition where two cationic monomers are needed for every one anionic monomer, or a 1:2 ratio (Figure 1d, blue curve). This condition of charge neutrality identifies the point where the maximum number of polymer chains will be incorporated into the coacervate phase; complexation will still occur at off stoichiometric conditions, but with a decreased level of coacervation. From a theoretical perspective, the composition of the bulk coacervate phase should be the same, with the difference being purely one of yield. However, experimentally, the preparation of off-stoichiometry dispersions of coacervate droplets can result in the recruitment of excess polymer to the surface of the droplet, imparting colloidal stability. ${ }^{20,21}$

For a given ratio of polycation-to-polyanion, the phase diagram for coacervation is then shown as a one-dimensional binodal curve, typically as a function of salt and total polymer concentration (Figure 1c). This binodal curve represents the slice through the larger twodimensional phase space at constant polymer composition (i.e., the black line). Samples prepared at a composition within this two-phase region will phase separate into two liquid phases, the polymer-rich coacervate phase and the polymer-poor supernatant phase. The composition of the resulting coacervate and supernatant phases is defined by the tie-line that connects these two points. One interesting (and potentially unintuitive) result of this phase behavior is that increasing the amount of polymer present in the initial sample mixture will not result in a commensurate increase in the polymer concentration in the resulting coacervate phase. Instead, samples prepared at different points along the tie-line will result in samples with different quantities of the same coacervate phase, as defined by the lever rule. Samples prepared at higher polymer concentrations will result in a larger coacervate volume, and vice versa.

There are numerous strategies for weakening or completely overcoming the interactions that cause complex coacervation. For example, changes in the solution $\mathrm{pH}$ or the addition of an excess of one of the polymers can shift the solution conditions outside of the two-phase window. $^{25,26}$ As suggested by Figure 1a-c, ionic strength is also an important variable for controlling coacervation. ${ }^{10,11,20}$ The addition of salt can facilitate screening of the electrostatic interactions and reduces the entropic gains associated with complexation. ${ }^{20,27}$ However, it is important to define the conditions for which this salt dissolution is being defined. As shown in Figure 1c, the critical point is the highest salt concentration for which phase separation can be observed. However, most experiments do not operate near the critical condition and are therefore interested in determining the concentration of salt above which phase separation is no longer observed for a given sample condition. This salt concentration is typically referred to as the salt resistance, ${ }^{28,29}$ and is dependent upon the choice of polymers, the coacervate composition, and the identity of the salt used. As was discussed regarding the effects of polymer concentration on coacervate composition, the salt resistance for any sample prepared along a given tie-line will be the same. However because tie-lines for coacervation tend to be non-horizontal (i.e., there is preferential partitioning of salt out of the coacervate phase), it is possible to change the salt resistance by changing polymer concentration, although this usually requires a significant change so as to move off of one tie-line and onto another. ${ }^{30}$ In the context of encapsulation studies, the salt resistance is an important parameter because it determines the concentration of salt required to dismantle the coacervate.

This introduction is intended to provide a foundational understanding of coacervate phase 
behavior to facilitate the use of coacervation for protein encapsulation. Thus far, our discussion has focused on complex coacervates formed from two species, a polycation and a polyanion. While the addition of protein does not alter these design rules, the chemical complexity of proteins can make interpretation of experimental data more challenging. For instance, most proteins carry a mixture of positive and negative charges. While electrostatic intuition would dictate that we consider only the net charge of a protein, there are examples where clustering of charges has allowed for complex coacervation to occur "on the wrong side of the isoelectric point," such that it would appear that complexation is occurring between two species of the same charge. ${ }^{31-34}$ It is also common to explore coacervation between a protein and two oppositely charged polymers, particularly for cases where the protein of interest is only weakly charged. $10,11,35,36$ In these cases it is necessary to consider the net charge of all three species, and to carefully balance the ionic strength of the system, as changes in the salt concentration will disfavor the incorporation of the more weakly charged protein in favor of stronger electrostatic interactions between the two more strongly charged polymers.

In the following sections we will discuss experimental strategies to characterize the coacervation phase behavior of a system of two oppositely charged polymers containing a protein cargo, as well as the incorporation of protein into the coacervate phase. These methods are intended to be general, and can be adapted to fit specific situations, such as the complexation of a protein with only a single, oppositely charged polymer.

\section{Materials, Equipment, and Reagents}

The materials required for these experiments include two oppositely charged polyelectrolytes, a protein of interest, salt, buffer (if desired), and acid/base for $\mathrm{pH}$ adjustments. We recommend that all solutions of polymer, protein, buffer (if desired) and salt be adjusted to the same $\mathrm{pH}$. Coomassie Brilliant Blue G-250 dye can be purchased alone or as part of a Bradford Assay kit.

Samples will be prepared via pipetting in microcentrifuge tubes and transferred into well plates (96- or 384-well plates are common. We recommend the use of a vortex mixer during sample preparation, an optical microscope for sample visualization, and a plate reader with UV/vis spectrophotometry capabilities. It is also possible to perform samples in cuvettes using a UV/vis spectrophotometer. Turbidity measurements are typically performed at a wavelength of light in the middle of the visible spectrum, and away from the absorbance peak of any of the materials $(\sim 562 \mathrm{~nm}$ is common). For quantifying the concentration of protein present, the Coomassie dye used in the Bradford assay is analyzed at a wavelength of $595 \mathrm{~nm}$, and the experiment can be performed in a well plate, cuvette, or other small volume UV/vis setup (e.g., NanoDrop). The use of absorbance at $280 \mathrm{~nm}$ requires the use of either UV-compatible cuvettes or a NanoDrop-type setup.

\section{Safety Considerations}

These techniques are safe to perform in a standard laboratory setting with the use of appropriate personal protection equipment such as safety glasses, lab coats, and gloves. It is recommended that experiments involving Coomassie dye be performed inside a chemical fume hood. For specific materials, refer to the safety data sheets.

To keep solutions sterile and dust free, keep the lids on all reagents, removing the lid only when pipetting, placing the cap back each time, though the lid may remain loose. Similarly, we 
recommend keeping all microcentrifuge tubes closed except when adding solution or transferring samples. The presence of dust can alter turbidity results. Care should also be taken to avoid cross-contamination of samples from pipette tips.

\section{Protocols}

\section{Characterizing Coacervate Phase Behavior}

While the ultimate goal of an experiment might be the encapsulation of a target protein, we recommend first characterizing the phase behavior of your coacervate system in terms of charge stoichiometry and salt resistance. These information will help in the planning of experiments related to protein encapsulation and will facilitate the interpretation of the resulting protein encapsulation data. These experiments are typically performed at relatively low concentrations of protein and polymer to limit reagent requirements. All experiments can be scaled up in terms of volumes and/or concentrations, though it is important to ensure that all samples are fully mixed and equilibrated.

\section{Polymer-Polymer Stoichiometry Experiments}

Stoichiometry experiments examine coacervate formation as a function of the ratio of polycation to polyanion at constant polymer concentration. Thus, while polymer stock solutions can be prepared at any concentration, we recommend the use of ionizable monomer concentration on a molar basis. The use of monomer concentration circumvents issues with polymer polydispersity, and allows for the easy analysis of results in terms of the stoichiometry of electrostatic interactions. A stock solution concentration of $10 \mathrm{mM}$ monomer is generally sufficient for turbidity experiments.

A typical stoichiometry experiment will span the range of possible charge fractions, which we will express in terms of the mole fraction of ionizable monomers of the polycation present in our sample, to observe both a peak in the data, and clear baselines. The data in Figures 3 and 4 and the experimental recipes listed in Tables 1 and 2 span the range of 0.1 to 0.9 . These data points can be equally spaced for initial experiments. However, once the location of the turbidity peak is known (or if its location is estimated based on the charge state of the polymers), it is useful to sample the concentration space around the peak more closely. Additionally, it is important to ensure that the final polymer concentration in the prepared samples results in a sufficiently high level of turbidity. This signal should be distinguishable from that of a blank solution at the same salt/buffer concentration, but in the absence of polymer. In our experience, samples should be prepared at a final concentration of at least $1 \mathrm{mM}$ (with respect to the total number of monomers), though this threshold concentration is a function of the path length through the sample.

To facilitate the preparation of fully equilibrated coacervate samples, we recommend that any salt, buffer, and excess water are combined first, followed by the addition of one of the polymers. It is then important to ensure that the sample is well mixed (e.g., vortexing for 5-10 s) before the second polymer is added, and that the sample is mixed again after the addition of the second polymer. It is also possible to prepare the polymer stock solutions at a specified concentration of salt/buffer, thereby eliminating the need to add these components separately, though this approach is less flexible in terms of adjusting experimental parameters. Once samples have been prepared, they should be mixed well and transferred to a well plate or cuvette for turbidity analysis. An example turbidity result for a stoichiometry experiment between poly $(L$-lysine $)$ and 
$\operatorname{poly}(D, L$-glutamate), degree of polymerization $\mathrm{N}=50, \mathrm{pH}=7.0$ is shown in Figure $2 \mathrm{a}$. The coacervate samples, as formed, should be a dispersion of droplets that gives the sample a cloudy and possibly opalescent appearance. At this point, the time between sample preparation and analysis is an important consideration, as the coacervate droplets can coalesce and settle over time and thus give variable turbidity readings. Generally, the turbidity signal should not be sensitive to differences of a few minutes, though this time scale can vary significantly based on the identity of the polymer system and the solution conditions.

Table 1: Sample preparation for complexation between poly $(L$-lysine $)\left(\mathrm{K}_{50}\right)$ and poly $(D, L$-glutamate $)\left(\mathrm{E}_{50}\right)$, degree of polymerization $\mathrm{N}=50, \mathrm{pH}=7.0$. Components were added from right to left as outlined in the protocol. The final monomer concentration is $1 \mathrm{mM}$.

\begin{tabular}{c|c|c|c|c} 
& $\begin{array}{c}\text { Charge } \\
\text { Fraction } \\
\mathbf{K}_{\mathbf{5 0}}(+)\end{array}$ & $\begin{array}{c}\text { Volume } \\
\mathbf{1 0 m M} \\
\mathbf{E}_{\mathbf{5 0}}(-) \\
(\boldsymbol{\mu L})\end{array}$ & $\begin{array}{c}\text { Volume } \\
\mathbf{1 0 m M} \\
\mathbf{K}_{\mathbf{5 0}}(+) \\
(\boldsymbol{\mu L})\end{array}$ & $\begin{array}{c}\text { Volume } \\
\text { Water } \\
\text { Added } \\
(\boldsymbol{\mu} \mathbf{L})\end{array}$ \\
\hline $\mathbf{1}$ & 0.100 & 10.8 & 1.2 & 108.0 \\
$\mathbf{2}$ & 0.200 & 9.6 & 2.4 & 108.0 \\
$\mathbf{3}$ & 0.300 & 8.4 & 3.6 & 108.0 \\
$\mathbf{4}$ & 0.400 & 7.2 & 4.8 & 108.0 \\
$\mathbf{5}$ & 0.425 & 6.9 & 5.1 & 108.0 \\
$\mathbf{6}$ & 0.450 & 6.6 & 5.4 & 108.0 \\
$\mathbf{7}$ & 0.475 & 6.3 & 5.7 & 108.0 \\
$\mathbf{8}$ & 0.500 & 6.0 & 6.0 & 108.0 \\
$\mathbf{9}$ & 0.525 & 5.7 & 6.3 & 108.0 \\
$\mathbf{1 0}$ & 0.550 & 5.4 & 6.6 & 108.0 \\
$\mathbf{1 1}$ & 0.575 & 5.1 & 6.9 & 108.0 \\
$\mathbf{1 2}$ & 0.600 & 4.8 & 7.2 & 108.0 \\
$\mathbf{1 3}$ & 0.700 & 3.6 & 8.4 & 108.0 \\
$\mathbf{1 4}$ & 0.800 & 2.4 & 9.6 & 108.0 \\
$\mathbf{1 5}$ & 0.900 & 1.2 & 10.8 & 108.0 \\
Blank & -- & 0.0 & 0.0 & 120.0 \\
\hline
\end{tabular}




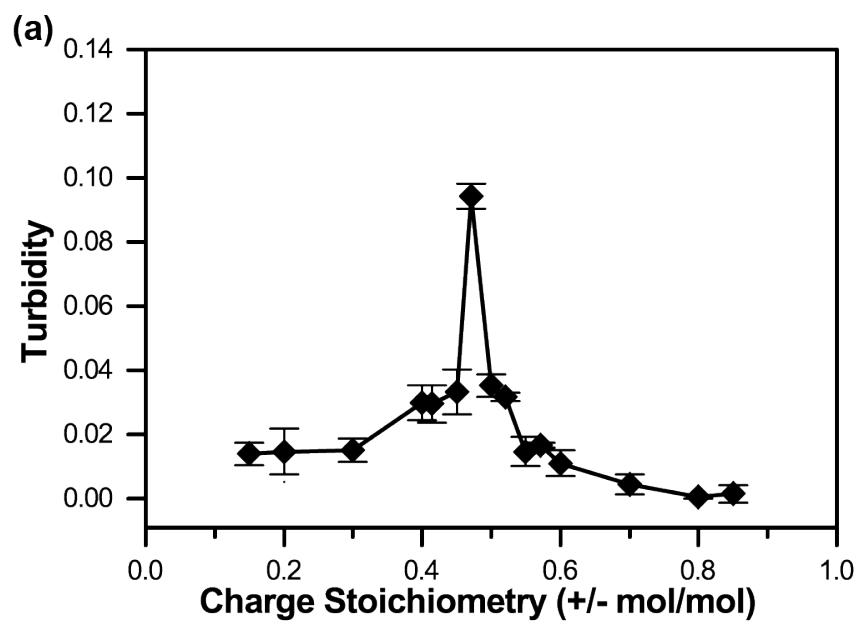

(b)

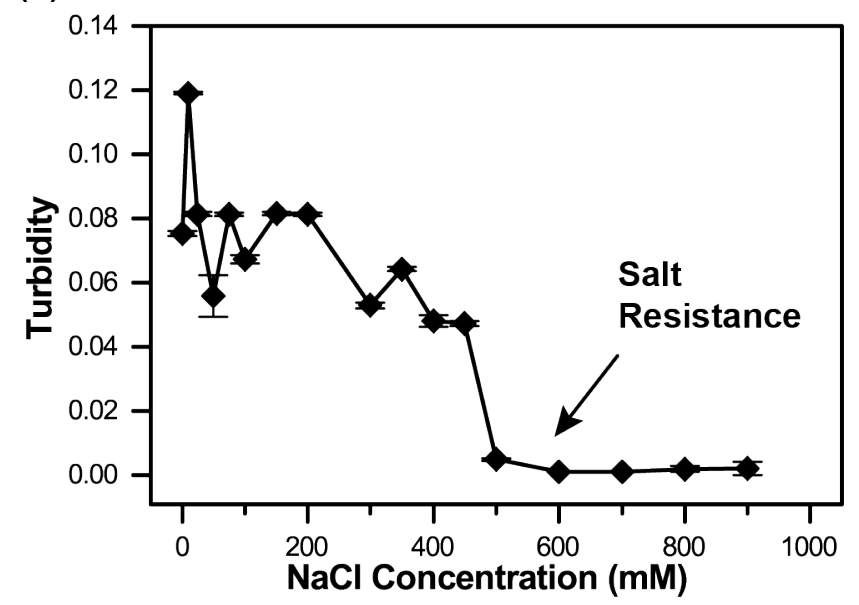

Figure 2: Turbidity data from (a) stoichiometry experiments involving poly $(L$-lysine $)$ and poly $(D, L$-glutamate $)$ with a degree of polymerization $\mathrm{N}=50, \mathrm{pH} 7.0$ with no added buffer and (b) salt resistance experiments as a function of increasing $\mathrm{NaCl}$ concentration. The final monomer concentration was $1 \mathrm{mM}$. Lines connecting the data points are a guide for the eye.

While all experimental results should be replicated in order to ensure reproducibility (i.e. "technical" replicates), we also recommend that samples be prepared in such a way that each sample can be split and analyzed separately (a repeat measurement of the sample, similar to a "biological" replicate). The use of three replicates/repeats will allow for the statistical analysis of the resulting data. In terms of sample preparation, the total volume of sample prepared for a repeated measurement should be $>3 x$ the volume needed for the three individual samples. For example, $35 \mu \mathrm{L}$ of sample is needed for a turbidity measurement using a 384-well plate. However, to ensure that sufficient sample volume is available for pipetting, a total sample volume of $120 \mu \mathrm{L}$ might be prepared.

After turbidimetry, samples should be inspected visually using an optical microscope. Standard brightfield microscopy with a 40x objective is typically sufficient for this procedure, though more advanced techniques such as phase contrast or differential interference contrast (DIC) can enhance the ability to visualize samples. For samples prepared at low polymer concentration, it may be difficult to distinguish very small droplets. The size of these coacervate droplets can be increased either by increasing the polymer concentration present in the sample, or by allowing the sample more time for the droplets to coalesce. The main goals in visualizing coacervate 
samples are to confirm the liquid vs. solid nature of the resulting materials, and provide secondary confirmation of trends (e.g., the presence or absence of coacervates) suggested via turbidity. Coacervate droplets should appear as circular/spherical structures either floating in solution or adhered onto a surface, whereas solid precipitation typically appears as fractal aggregates (Figure 3).
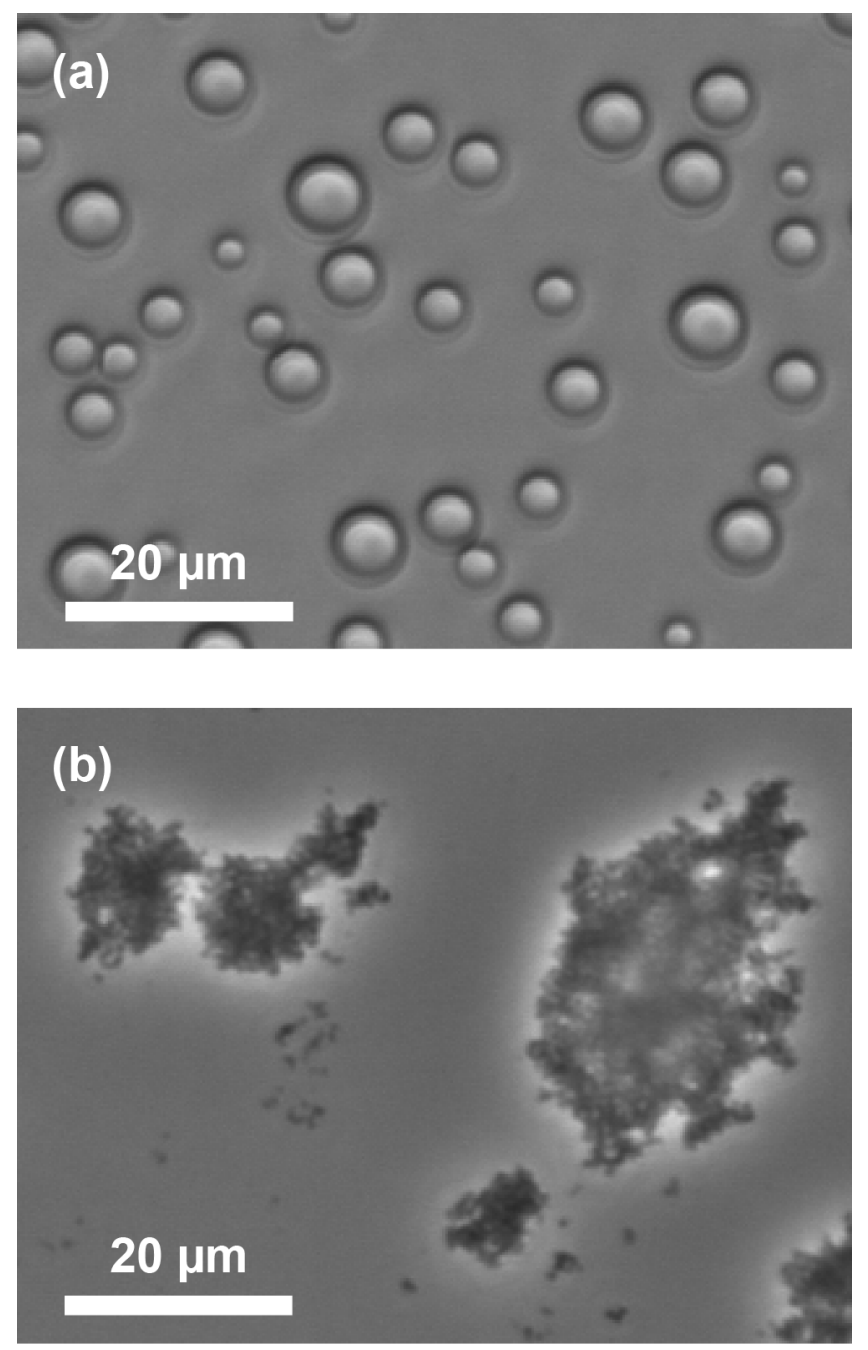

Figure 3: Optical micrographs of (a) liquid complex coacervate droplets of poly $(L$-lysine) and poly $(D, L$-glutamate), and (b) fractal solid precipitates resulting from the interaction of poly( $L$-lysine $)$ and poly( $L$-glutamate). All samples were prepared using polymers with degree of polymerization $\mathrm{N}=100$ at a total monomer concentration of $6 \mathrm{mM}$ in $100 \mathrm{mM} \mathrm{NaCl}, \mathrm{pH} 7.0$.

An important consideration in the preparation of coacervates is whether they are fully equilibrated. This question can be answered by testing whether or not the results of an experiment are sensitive to the order of polymer addition.

\section{Polymer-Polymer Salt Resistance Experiments}

The goal of salt resistance experiments is to identify the concentration of salt (for a given total polymer concentration and stoichiometric ratio) above which phase separation is no longer observed. This provides information on the location of the binodal curve, and informs the design of experimental procedures where dissolution of the coacervate phase is needed. The magnitude 
of the salt resistance is dependent upon the length and identity of the polymers, the choice of salt, the solution $\mathrm{pH}$, etc. Generally speaking, increases in polymer length, hydrophobicity, and charge density will result in higher values of the salt resistance at the same polymer concentration.

In designing a salt resistance experiment for a new polymer, it may be necessary to perform a screening-level experiment to identify the general range of salt concentrations over which the experiment should be performed. Generally, the salt resistance can be identified as a clear decrease in the turbidity signal with increasing salt concentration. However, it is useful to combine turbidimetry measurements with direct visualization of the samples via optical microscopy to confirm results. An example of salt resistance data for the system of poly $(L-$ lysine) and poly $(D, L$-glutamate $)$ degree of polymerization $\mathrm{N}=50, \mathrm{pH}=7.0$ is shown in Figure $2 \mathrm{~b}$.

A general step-by-step protocol for these polymer-only experiments is given below.

\section{Experimental Protocol for Coacervate Samples (no protein)}

1. Set up microcentrifuge tubes for each sample and a blank, labeling appropriately.

2. Pipette the appropriate amount of water into each tube (as needed).

3. Pipette the appropriate amount of buffer solution into each tube (as needed).

4. Pipette the appropriate amount of salt solution into each tube (as needed).

5. Pipette the appropriate amount of the first polyelectrolyte solution into each tube.

6. Vortex each tube for 5-10 s.

7. Pipette the appropriate amount of the second polyelectrolyte solution into each tube, vortexing for 5-10 s immediately after each addition.

8. Transfer aliquots of each sample to the well plate or cuvette for turbidity analysis.

9. Inspect each sample via optical microscopy.

Table 2: Sample preparation for a salt curve for between poly $\left(L\right.$-lysine) $\left(\mathrm{K}_{50}\right)$ and poly $(D, L$-glutamate $)\left(\mathrm{E}_{50}\right)$, degree of polymerization $\mathrm{N}=50$, in $10 \mathrm{mM}$ HEPES buffer $\mathrm{pH}=7.0$. Components were added from right to left as outlined in the protocol. The final monomer concentration is $1 \mathrm{mM}$.

\begin{tabular}{c|c|c|c|c|c} 
& $\begin{array}{c}\text { Charge } \\
\text { Fraction } \\
\mathbf{K}_{\mathbf{5 0}}(+)\end{array}$ & $\begin{array}{c}\text { Volume } \\
\mathbf{1 0 m M} \\
\mathbf{E}_{\mathbf{5 0}}(-) \\
(\boldsymbol{\mu L})\end{array}$ & $\begin{array}{c}\text { Volume } \\
\mathbf{1 0 m M} \\
\mathbf{K}_{\mathbf{5 0}}(+) \\
(\boldsymbol{\mu L})\end{array}$ & $\begin{array}{c}\text { Volume } \\
\mathbf{2} \mathbf{M} \\
\mathbf{N a C l} \\
\text { Added } \\
(\boldsymbol{\mu L})\end{array}$ & $\begin{array}{c}\text { Volume } \\
\text { Water } \\
\text { Added } \\
(\boldsymbol{\mu L} \mathbf{L})\end{array}$ \\
\hline $\mathbf{1}$ & 0.500 & 6.0 & 6.0 & 0.0 & 108.0 \\
$\mathbf{2}$ & 0.500 & 6.0 & 6.0 & 1.5 & 106.5 \\
$\mathbf{3}$ & 0.500 & 6.0 & 6.0 & 3.0 & 105.0 \\
$\mathbf{4}$ & 0.500 & 6.0 & 6.0 & 4.5 & 103.5 \\
$\mathbf{5}$ & 0.500 & 6.0 & 6.0 & 6.0 & 102.0 \\
$\mathbf{6}$ & 0.500 & 6.0 & 6.0 & 9.0 & 99.0 \\
$\mathbf{7}$ & 0.500 & 6.0 & 6.0 & 12.0 & 96.0 \\
$\mathbf{8}$ & 0.500 & 6.0 & 6.0 & 18.0 & 90.0 \\
$\mathbf{9}$ & 0.500 & 6.0 & 6.0 & 21.0 & 87.0 \\
$\mathbf{1 0}$ & 0.500 & 6.0 & 6.0 & 24.0 & 84.0 \\
$\mathbf{1 1}$ & 0.500 & 6.0 & 6.0 & 27.0 & 81.0 \\
$\mathbf{1 2}$ & 0.500 & 6.0 & 6.0 & 30.0 & 78.0 \\
$\mathbf{1 3}$ & 0.500 & 6.0 & 6.0 & 36.0 & 72.0 \\
$\mathbf{1 4}$ & 0.500 & 6.0 & 6.0 & 42.0 & 66.0
\end{tabular}




\begin{tabular}{c|c|c|c|c|c}
$\mathbf{1 5}$ & 0.500 & 6.0 & 6.0 & 48.0 & 60.0 \\
$\mathbf{1 6}$ & 0.500 & 6.0 & 6.0 & 54.0 & 54.0 \\
Blank & -- & 0.0 & 0.0 & 0.0 & 120.0 \\
\hline
\end{tabular}

\section{Polymer-Polymer-Protein Stoichiometry Experiments}

The design of a stoichiometry experiment for a system that combines protein in the presence of a polycation and polyanion varies only slightly in design and intent. While more extensive experiments that vary the relative amounts of each polymer and the quantity of protein can be performed to map out the complete phase behavior of this more complex system, simpler experiments that consider the effect of polymer charge stoichiometry in the presence of a constant level of protein can also be performed (see Table 3 for an example recipe). Here, we will discuss the design of these simpler experiments and how the addition of a protein as a third charged macromolecule can alter the phase behavior of the system.

Table 3: Sample preparation for complexation between poly $(L$-lysine $)\left(\mathrm{K}_{50}\right)$ and poly $(D, L$-glutamate $)\left(\mathrm{E}_{50}\right)$, degree of polymerization $\mathrm{N}=50$, in $10 \mathrm{mM}$ HEPES buffer $\mathrm{pH}=7.0$ with bovine serum albumin (BSA). Components were added from right to left as outlined in the protocol. The final monomer concentration is $7 \mathrm{mM}$ and the final protein concentration is 50 $\mu \mathrm{g} / \mathrm{mL}$.

\begin{tabular}{|c|c|c|c|c|c|c|}
\hline & $\begin{array}{c}\text { Charge } \\
\text { Fraction } \\
\mathbf{K}_{\mathbf{5 0}}(+)\end{array}$ & $\begin{array}{c}\text { Volume } \\
10 \mathrm{mM} \\
\mathbf{E}_{50}(-) \\
(\mu \mathrm{L})\end{array}$ & $\begin{array}{c}\text { Volume } \\
\mathbf{1 0 m M} \\
\mathbf{K}_{\mathbf{5 0}}(+) \\
(\mu \mathrm{L})\end{array}$ & $\begin{array}{c}\text { Volume } 2 \\
\text { mg/mL } \\
\text { BSA(-) } \\
\text { Added } \\
(\mu L)\end{array}$ & $\begin{array}{c}\text { Volume } \\
\text { 0.5 M } \\
\text { HEPES } \\
\text { Added } \\
(\mu L) \\
\end{array}$ & $\begin{array}{c}\text { Volume } \\
\text { Water } \\
\text { Added } \\
(\boldsymbol{\mu L})\end{array}$ \\
\hline 1 & 0.100 & 151.2 & 16.8 & 6.0 & 4.80 & 61.2 \\
\hline 2 & 0.200 & 134.4 & 33.6 & 6.0 & 4.80 & 61.2 \\
\hline 3 & 0.300 & 117.6 & 50.4 & 6.0 & 4.80 & 61.2 \\
\hline 4 & 0.400 & 100.8 & 67.2 & 6.0 & 4.80 & 61.2 \\
\hline 5 & 0.425 & 96.6 & 71.4 & 6.0 & 4.80 & 61.2 \\
\hline 6 & 0.450 & 92.4 & 75.6 & 6.0 & 4.80 & 61.2 \\
\hline 7 & 0.475 & 88.2 & 79.8 & 6.0 & 4.80 & 61.2 \\
\hline 8 & 0.500 & 84.0 & 84.0 & 6.0 & 4.80 & 61.2 \\
\hline 9 & 0.525 & 79.8 & 88.2 & 6.0 & 4.80 & 61.2 \\
\hline 10 & 0.550 & 75.6 & 92.4 & 6.0 & 4.80 & 61.2 \\
\hline 11 & 0.575 & 71.4 & 96.6 & 6.0 & 4.80 & 61.2 \\
\hline 12 & 0.600 & 67.2 & 100.8 & 6.0 & 4.80 & 61.2 \\
\hline 13 & 0.700 & 50.4 & 117.6 & 6.0 & 4.80 & 61.2 \\
\hline 14 & 0.800 & 33.6 & 134.4 & 6.0 & 4.80 & 61.2 \\
\hline 15 & 0.900 & 16.8 & 151.2 & 6.0 & 4.80 & 61.2 \\
\hline Blank & 0.000 & 0.0 & 0.0 & 0.0 & 4.80 & 235.2 \\
\hline
\end{tabular}

Our initial stoichiometry experiments with just the two-polymer system served to identify the composition corresponding to maximum coacervate yield, corresponding to a charge-neutral mixture of the two polymers. However, the addition of a charged protein would be expected to shift this optimal condition. As can be seen in Figure 4a, the addition of negatively-charged bovine serum albumin (BSA) to a mixture of poly( $L$-lysine) and poly $(D, L$-glutamate) degree of polymerization $\mathrm{N}=50$, in $10 \mathrm{mM}$ HEPES, $\mathrm{pH}=7.0$ results in a shift in the resulting turbidity signal to higher mole fractions of the polycation. A shift in the opposite direction, to "net negative" conditions would be expected for positively-charged proteins.

As for the two-polymer system, it is important to determine whether the order of mixing has any 
effect on the resulting coacervates.

\section{Polymer-Polymer-Protein Salt Resistance Experiments}

Salt resistance experiments can also be performed for samples including proteins. However, most proteins would be expected to have a lower charge content and charge density than the associated polymers. As such, it is a reasonable assumption that the salt resistance of the threemacromolecule system should be lower than that of the polymer-only system.
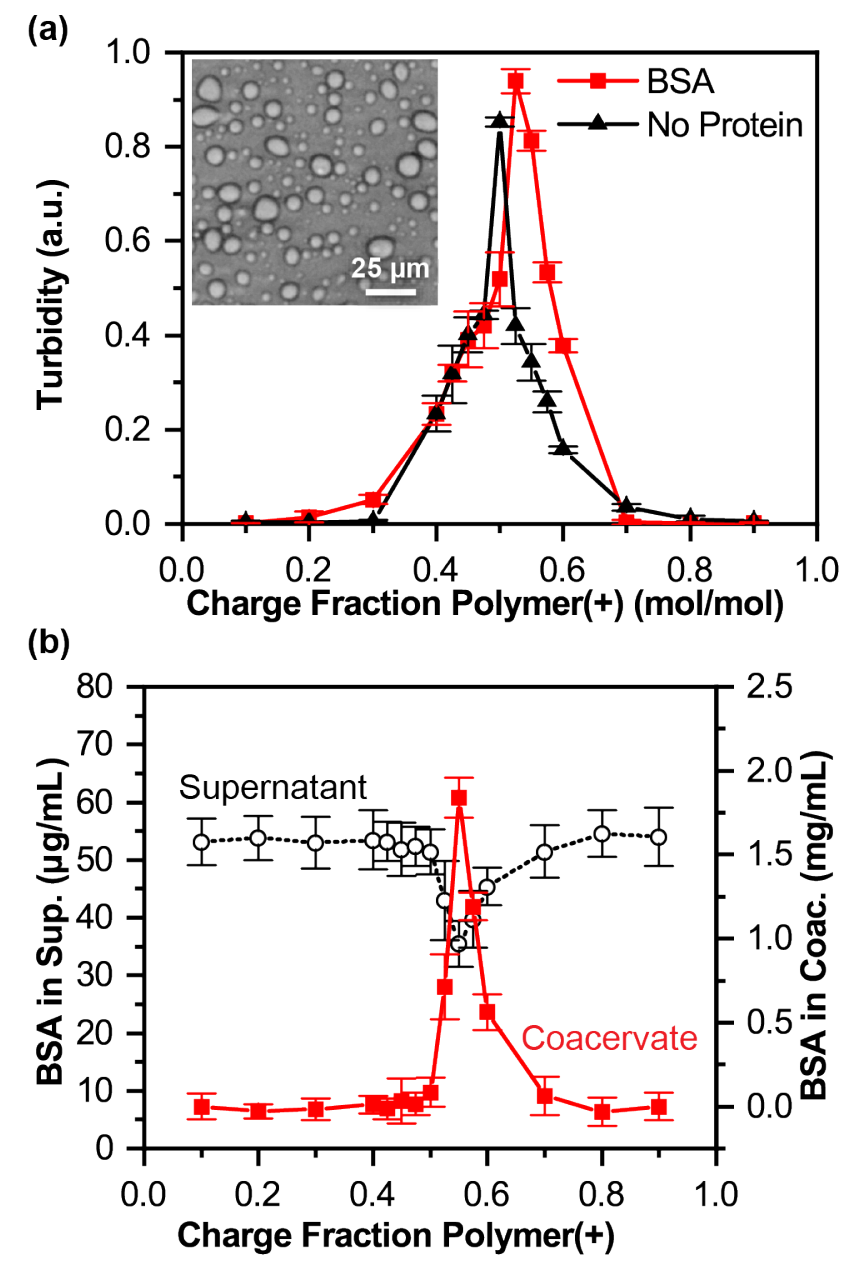

Figure 4: (a) Turbidity data from stoichiometry experiments involving poly $(L$-lysine $)$ and poly $(D, L$-glutamate $)$ with a degree of polymerization $\mathrm{N}=50$ (black) and for the same system with the anionic protein bovine serum albumin (BSA) (red) in $10 \mathrm{mM}$ HEPES. The inset optical micrograph depicts the formation of coacervate droplets with BSA. (b) The corresponding concentration of protein in the supernatant (black) and coacervate (red) phases, as determined using a Bradford assay. (Blocher McTigue, W. C.; Perry, S. L. Design Rules for Encapsulating Proteins into Complex Coacervates. Soft Matter 2019, 15, 30893103. Reproduced by permission of The Royal Society of Chemistry. Ref. [35].)

A general step-by-step protocol for these polymer-polymer-protein experiments is given below.

\section{Experimental Protocol for Coacervate Samples (with protein)}

1. Set up microcentrifuge tubes for each sample and a blank, labeling appropriately.

2. Pipette the appropriate amount of water into each tube (as needed).

3. Pipette the appropriate amount of buffer solution into each tube (as needed).

4. Pipette the appropriate amount of salt solution into each tube (as needed). 
5. Pipette the appropriate amount of the first polyelectrolyte solution into each tube.

6. Vortex each tube for 5-10 s.

7. Pipette the appropriate amount of the protein solution into each tube, vortexing for 5-10 s immediately after each addition.

8. Pipette the appropriate amount of the second polyelectrolyte solution into each tube, vortexing for 5-10 s immediately after each addition.

9. Transfer aliquots of each sample to the well plate or cuvette for turbidity analysis.

10. Inspect each sample via optical microscopy.

\section{Quantifying Protein Incorporation into Complex Coacervates}

While turbidity and optical microscopy can be used to determine whether complexation has occurred or not, these measurements do not provide information on the incorporation of protein into the coacervate phase. Instead, separate measures of the protein concentration in the coacervate and supernatant phases must be made, along with a determination of the volume of each phase.

Once the concentrations for both the coacervate and supernatant phases are known, several other parameters may be determined. The first is the encapsulation efficiency $(E E)$ with is the percentage of cargo (by mass) sequestered, in this case, by complex coacervates. Measuring the supernatant volume and multiplying by the concentration of protein in the supernatant phase gives the mass of cargo in the supernatant. Subtracting this mass from the total mass of protein added to the system during sample preparation allows for calculation of the mass of protein in the coacervate phase. These masses can then calculate the encapsulation efficiency:

$$
E E=\frac{m_{\text {coac. protein }}}{m_{\text {total protein }}} \times 100 \%
$$

A partition coefficient, on the other hand, is typically defined as the ratio of the concentration of protein in the dense coacervate phase over the concentration of the cargo in the dilute phase, written as:

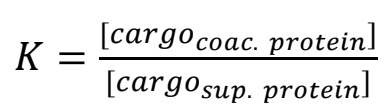

Finally, loading or loading capacity is the amount of protein in the coacervate compared to the total mass of the coacervate, i.e., it describes what fraction of the coacervate phase is comprised of the cargo:

$$
L C=\frac{m_{\text {coac. protien }}}{m_{\text {coac. } \text { total }}} \times 100 \%
$$

Below, we will detail strategies for assaying the protein concentration in coacervate samples using a colorimetric Bradford assay, and via direct measurement of protein absorbance.

\section{Bradford Assay}

The protein quantification experiments described here are intended to be run in parallel with a stoichiometry-type experiment, as described above, with half of the total sample volume used for turbidity experiments, and the other half used for protein quantification. As in the case of turbidity experiments, it is important to measure both repeat and replicate samples in order to 
allow for statistical analysis of the results.

The Coomassie brilliant blue G-250 dye used in the Bradford assay is typically described as interacting with basic amino acids in hydrophobic pockets, but primarily responds to arginine residues, as well as histidine, tryptophan, tyrosine, and phenylalanine to a lesser extent. ${ }^{38-40}$ Typically, users buy a kit that may come with protein standards (e.g. bovine serum albumin, BSA) to aid in the creation of a calibration curve. However, individual proteins interact differently with the Coomassie dye, based on their amino acid sequence. Therefore, we recommend creating calibration curves directly with the protein of interest. Furthermore, all calibration curves must be prepared at the solution conditions (i.e., $\mathrm{pH}$, salt concentration, etc.) expected in the final sample. One consequence of this is that separate calibration curves are required for supernatant samples, which can be measured directly, and coacervate samples, which must be performed at a higher salt concentration in order to dismantle the coacervate (Figure 5).

In addition to considering the specific interactions of the Coomassie dye with the protein at different solution conditions, it is critical to determine the potential background signal that might result from the dye interacting with the polymers used in the coacervate. While the interaction of the dye with poly $(L$-lysine $)$ and $\operatorname{poly}(D, L$-glutamate $)$ has been shown to be minimal, ${ }^{35}$ much stronger interactions occur when more hydrophobic polymers such as poly(styrene sulfonate) (PSS), poly(diallyldimethylammonium chloride) (PDADMAC), and methacrylate-based polyelectrolytes. The interaction between polymer and dye can be tested by mixing a 1:1 volume ratio of the dye with a solution of polymer at the concentration intended for use in experiments. Qualitatively, a strong interaction between the dye and the polymers can be observed visually as a color change from brackish-brown to blue. Quantitatively, it is important to consider whether the level of background signal resulting from the polymers in the coacervate will swamp the potential absorbance signal from the protein by itself. This comparison can be done via an absorbance measurement at $595 \mathrm{~nm}$. While it is possible to perform a background subtraction to account for signal associated with the polyelectrolytes, this correction can become complicated if the concentration of each of the polymers varies across different samples.

The linear range of the Bradford assay is a function of the specific interaction of the protein with the Coomassie dye, and the volumetric scale (and thus the absorbance path length) at which the experiment is performed. Different scale protocols have been developed for the Bradford assay depending on the desired sample volumes and the range of concentrations. The main difference in the protocols is the ratio of Coomassie Dye solution to protein sample. For example, the "standard" Bradford protocol uses a 50:1 ratio of dye to sample, and has a typical range of $\sim 125-$ $1,000 \mu \mathrm{g} / \mathrm{mL}$. In contrast, the "micro assay" uses a 1:1 ratio of dye to sample and has a linear range of approximately $1-10 \mu \mathrm{g} / \mathrm{mL}{ }^{39}$ These ratios, as well as the sample path length can be tuned to optimize a protocol for a specific protein target and/or solution condition. For more information on the Bradford assay, we encourage readers to refer to Refs. [38,39].

For the example data shown in Figure 4 and Table 3, we assayed the uptake of bovine serum albumin (BSA) into coacervates formed from poly $(L$-lysine $)$ and poly $(D, L$-glutamate $)$ with a degree of polymerization $\mathrm{N}=50$, in $10 \mathrm{mM}$ HEPES, $\mathrm{pH}$ 7.0. The experiment tested the incorporation of $50 \mu \mathrm{g} / \mathrm{mL}$ BSA as a function of the charge stoichiometry of the two polymers. A "micro assay" style Bradford assay was used to quantify the protein concentration in both the 
coacervate and the supernatant phases. Samples were prepared at a total volume of $240 \mu \mathrm{L}, 105$ $\mu \mathrm{L}$ of which was used for turbidity measurements (3 repeat samples of $35 \mu \mathrm{L}$ each), and $115 \mu \mathrm{L}$ of which was used for protein quantification. After separating the coacervate and supernatant, protocols were developed separately for the two phases.

Having measured the total volume of the supernatant for each sample to facilitate future mass balance calculations, $115 \mu \mathrm{L}$ of supernatant was then aliquotted into a clean microcentrifuge tube and combined with an equal volume $(115 \mu \mathrm{L})$ of $1 x$ Coomassie dye. Three repeat aliquots of 35 $\mu \mathrm{L}$ each were then pipetted into a 384-well plate for absorbance measurements. The absorbance data were converted to concentration values using a standard curve with samples prepared in 10 mM HEPES, $\mathrm{pH} 7.0$.

For the coacervate samples, visual inspection after centrifugation had determined that the volume of coacervate was very small $(\sim 0-2.2 \mu \mathrm{L})$. We then added $70 \mu \mathrm{L}$ of $2.0 \mathrm{M} \mathrm{NaCl}$ to disassemble the coacervate. This sample was then mixed with an equal volume $(70 \mu \mathrm{L})$ of $1 \mathrm{x}$ Coomassie dye. For these samples, we chose to neglect the volume of our coacervate in subsequent concentration calculations as an error of $\sim 0-2.2 \mu \mathrm{L}$ represents a $\sim 3 \%$ volume error. Three repeat aliquots of $35 \mu \mathrm{L}$ each were then pipetted into a 384-well plate for absorbance measurements, as above. The absorbance data for coacervate samples were converted to concentration using a standard curve with samples prepared at a concentration of $2 \mathrm{M} \mathrm{NaCl}$.

The data in Figure 4a show a strong peak in the turbidity at a mole fraction of polycation of approximately 0.525 . This peak in the turbidity data corresponds directly with a strong increase in the concentration of protein present in the coacervate phase (to nearly $2.0 \mathrm{mg} / \mathrm{mL}$, or 2,000 $\mu \mathrm{g} / \mathrm{mL}$ ), and a corresponding decrease in the protein from the supernatant (Figure $4 \mathrm{~b}$ ). In contrast, for samples at the extremes of the stoichiometric range where no phase separation occurred, we recovered the expected value of $50 \mu \mathrm{g} / \mathrm{mL}$ BSA that was input into the system.

\section{Experimental Protocol for Protein Quantification Using the Bradford Assay}

1. Perform steps 1-10 as described above for "Experimental Protocol for Coacervate Samples (with protein)." Samples should be prepared at a volume scale 2x the quantity needed for a turbidity experiment.

2. Centrifuge samples to phase separate the coacervate and supernatant phases for $20 \mathrm{~min}$ at $14,000 \mathrm{rpm}(18,800 \mathrm{xg})$ at $15^{\circ} \mathrm{C}$.

3. Use a pipette to transfer the supernatant into a new microcentrifuge tube carefully, noting the volume.

* In many instances, such as the samples described in Table 3 , it is difficult to obtain accurate measurements of coacervate volumes that can be on the order of $\sim 1 \mu \mathrm{L}$. To circumvent this issue, we can estimate this volume by subtracting the measured value of the supernatant volume from the total sample volume.

4. Pipette a sufficient volume of a concentrated stock solution of salt (e.g., $2 \mathrm{M} \mathrm{NaCl})$ to cause the dissolution of the coacervate phase and create a large enough volume sample to run multiple aliquots for the Bradford assay. The necessary concentration of salt can be determined by a salt resistance experiment, as described above.

5. Pipette the necessary volume of Coomassie dye to the supernatant and coacervate samples.

6. Vortex each tube for 5-10 s. 
7. Transfer aliquots of the supernatant and coacervate samples into to the well plate or cuvette, taking care to avoid and/or remove bubbles.

8. Measure the absorbance of the samples and a blank at $595 \mathrm{~nm}$.

9. Convert the measured absorbance values to protein concentration using the appropriate standard curve.

\section{Utilizing Absorbance at $280 \mathrm{~nm}$}

While the Bradford assay is performed using a colorimetric readout, measuring absorbance at $280 \mathrm{~nm}$ (A280) requires special consideration. Many standard well plates and disposable cuvettes may not be suited for this method as many plastics absorb in the UV. Specialized plastic cuvettes, quartz cuvettes, and small volume absorbance setups such as the NanoDrop or Take3 should be used for these measurements.

The linear range of concentrations measurable by absorbance at $280 \mathrm{~nm}$ generally extends from $\sim 20 \mu \mathrm{g} / \mathrm{mL}$ to $\sim 3,000 \mu \mathrm{g} / \mathrm{mL} .{ }^{41}$ However, the specific measurable range will be a function of the extinction coefficient for a given protein, in tandem with potential background interference from other components of the solution, as well as the path length of the sample. For example, the standard range for the small volume NanoDrop system is between $0.1 \mathrm{mg} / \mathrm{mL}$ to $400 \mathrm{mg} / \mathrm{mL}$ for BSA, which has an extinction coefficient of $43,824 \mathrm{M}^{-1} \mathrm{~cm}^{-1}$, assuming a molecular weight of $66.4 \mathrm{kDa}^{42}$ However, this range can be heavily influenced by the presence of other molecules present in solution, such as salt, buffer, surfactants, etc. Table 3 lists the maximum allowable concentration for a range of common chemicals related to these absorbance measurements.

In the specific context of complex coacervate samples, it is important to test whether or not the polyelectrolytes used to form the coacervate absorb at $280 \mathrm{~nm}$. While it is possible to perform a background subtraction to account for signal associated with the polyelectrolytes, this correction can become complicated if the concentration of each of the polymers varies across different samples.

In each case, there is a known path length to use for $l$ in Beer's equation:

$$
A=c \varepsilon l
$$

Additionally, the extinction coefficient used in the calculations will vary the calculated concentrations. This coefficient can be found using programs such as ProtParam or looking into the literature. ${ }^{43}$ If there is no known extinction coefficient and no sequence, a standard assumption is that an absorbance reading of 1.0 is equal to $1 \mathrm{mg} / \mathrm{mL}$ based on a $0.1 \%$ or 1 $\mathrm{mg} / \mathrm{mL}$ protein concentration producing an absorbance at $280 \mathrm{~nm}$ of 1.0 with a path length of 10 $\mathrm{mm}$ or $1 \mathrm{~cm} .{ }^{40}$

In general, the molar extinction coefficient (in units of $\mathrm{M}^{-1} \mathrm{~cm}^{-1}$ ) is approximated as:

$$
\varepsilon=5500 W+1490 Y+125 C
$$

where $W$ is the number of tryptophans, $Y$ is the number of tyrosines, and $C$ is the number of cysteines in the protein sequence. Each number before the amino acids is the molar absorptivity at $280 \mathrm{~nm}$ for that residue. It is noteworthy that extinction coefficients are frequently reported using a range of different units, thus knowing the conversions is sometimes necessary for 
analysis. To convert to a percent extinction coefficient, which has units of $(\mathrm{g} / 100 \mathrm{~mL})^{-1} \mathrm{~cm}^{-1}$, the formula is:

$$
\varepsilon_{1 \%}=\frac{10 \varepsilon}{M W}
$$

where $M W$ is the molecular weight of the protein. Another common form is the $0.1 \%$ extinction coefficient and has units of $\left(\mathrm{mg} / \mathrm{mL}^{-1} \mathrm{~cm}^{-1}\right.$. Converting to the $0.1 \%$ extinction coefficient follows:

$$
\varepsilon_{0.1 \%}=\frac{\varepsilon \%}{10}=\frac{\varepsilon}{M W}
$$

The use of a program to calculate protein concentration using absorbance at $280 \mathrm{~nm}$ may have correction calculations, appropriate reading of the literature and user guides will help determine such corrections. If it is suspected that the protein sample has nucleic contamination, Eq. 8 may be used for an approximate determination: ${ }^{38,40}$

$$
[\text { protein }](m g / m L)=\left(1.55 \times A_{280}\right)-\left(0.76 \times A_{260}\right)
$$

However, a potentially simpler method for protein quantification at $280 \mathrm{~nm}$ is to create a standard curve of the protein. This is useful for systems that employ various buffer conditions that may affect results and does not require a NanoDrop program or use of an extinction coefficient. Standard curves are made by preparing known sample concentrations at desired conditions and running them at $280 \mathrm{~nm}$, subtracting out a blank. It is good for scientists, however, to understand where the math comes from if a NanoDrop or similar system is used. Note that many of these systems have additional blank subtraction and correction procedures built in.

\section{Experimental Protocol for Protein Quantification Using A280}

1. Perform steps 1-4 as described above for the Bradford Protocol.

2. Pipette an aliquot of known volume for absorbance measurements.

a. A Take3 or NanoDrop system allow for the use of $\sim 2 \mu \mathrm{L}$ with a known path length.

b. A UV-compatible cuvette can be used with a larger sample volume.

3. Measure the absorbance of the samples and a blank at $280 \mathrm{~nm}$.

4. Convert the measured absorbance values to protein concentration using the appropriate standard curve.

5. Samples will be read at $280 \mathrm{~nm}$ via a well plate reader

a. Take3 / NanoDrop have built in programs for $280 \mathrm{~nm}$ and are recommended for specific moieties, such as BSA and IgG, with appropriate extinction coefficients

b. A standard curve may be used to determine protein concentration if extinction coefficient is unknown or the extinction coefficient can be calculated via code or from another program such as ProtParam. ${ }^{43}$

\section{Standard Curves}

For all of the methods described, it is necessary to create a standard curve to determine the concentration of protein from the measured absorbance signal. While BSA is commonly used as a protein standard to generate a "universal" set of standard curves, the colorimetric signal from the Coomassie dye and/or the absorbance at $280 \mathrm{~nm}$ can vary widely from protein to protein, and 
as a function of solution conditions. Thus, we advise the creation of a standard curve for each protein at every set of experimental conditions used. Table 4Error! Reference source not found. outlines limits for several common buffers, salts, etc. for the Bradford assay and $280 \mathrm{~nm}^{40}$ Generally speaking, measurements at $280 \mathrm{~nm}$ are sensitive to molecules that have double bonds between carbons or carbon and oxygen, ${ }^{40}$ while the Bradford assay does not tolerate high concentrations of detergents. ${ }^{38}$

Table 4: Concentration limits for protein assays using Coomassie dye and absorbance at $280 \mathrm{~nm}$. Values adapted from Olson and Markwell Assays for Determination of Protein Concentration. ${ }^{38}$

\begin{tabular}{|c|c|c|c|}
\hline \multicolumn{4}{|c|}{ Concentration Limits for Protein Assays ${ }^{a}$} \\
\hline Substance $^{b}$ & & Dye $^{c}$ & $280 \mathrm{~nm}^{\mathrm{d}}$ \\
\hline \multicolumn{4}{|c|}{ Acids and Bases } \\
\hline & $\mathrm{HCl}$ & $0.1 \mathrm{M}$ & $>1 \mathrm{M}$ \\
\hline & $\mathrm{NaOH}$ & $0.1 \mathrm{M}$ & $>1 \mathrm{M}$ \\
\hline & PCA & & $10 \%$ \\
\hline & TCA & & $10 \%$ \\
\hline \multicolumn{4}{|l|}{ Buffers } \\
\hline & Acetate & $0.6 \mathrm{M}$ & $0.1 \mathrm{M}$ \\
\hline & Ammonium sulfate & $1 \mathrm{M}$ & $>50 \%$ \\
\hline & Citrate & $50 \mathrm{mM}$ & $5 \%$ \\
\hline & Glycine & $0.1 \mathrm{M}$ & $1 \mathrm{M}$ \\
\hline & HEPES & $100 \mathrm{mM}$ & \\
\hline & Phosphate & $2 \mathrm{M}$ & $1 \mathrm{M}$ \\
\hline & Tris & $2 \mathrm{M}$ & $0.5 \mathrm{M}$ \\
\hline \multicolumn{4}{|l|}{ Detergents } \\
\hline & Brij 35 & & $1 \%$ \\
\hline & CHAPS & & $10 \%$ \\
\hline & Deoxycholate & $0.25 \%$ & $0.30 \%$ \\
\hline & Digitonin & & $10 \%$ \\
\hline & Lubrol PX & & $10 \%$ \\
\hline & Octylglucoside & & $10 \%$ \\
\hline & SDS & $0.10 \%$ & $0.10 \%$ \\
\hline & Triton X-100 & $0.10 \%$ & $0.02 \%$ \\
\hline & Triton $X-100(\mathrm{R})$ & & $>10 \%$ \\
\hline & Tween 20 & & $0.30 \%$ \\
\hline \multicolumn{4}{|l|}{ Reductants } \\
\hline & Dithiothreitol & $1 \mathrm{M}$ & $3 \mathrm{mM}$ \\
\hline & 2-Mercaptoethanol & $1 \mathrm{M}$ & $10 \mathrm{mM}$ \\
\hline \multicolumn{4}{|l|}{ Miscellaneous } \\
\hline & DNA/RNA & $0.25 \mathrm{mg}$ & $1 \mu \mathrm{g}$ \\
\hline & DMSO & & $20 \%$ \\
\hline & EDTA & $0.1 \mathrm{M}$ & $30 \mathrm{mM}$ \\
\hline & Glycerol & $100 \%$ & $40 \%$ \\
\hline
\end{tabular}




\begin{tabular}{l|l|l}
$\mathrm{KCl}$ & $1 \mathrm{M}$ & $100 \mathrm{mM}$ \\
$\mathrm{NaCl}$ & $5 \mathrm{M}$ & $>1 \mathrm{M}$ \\
Sucrose & $1 \mathrm{M}$ & $2 \mathrm{M}$ \\
Urea & $6 \mathrm{M}$ & $>1 \mathrm{M}$ \\
\hline
\end{tabular}

\footnotetext{
${ }^{a}$ This table is a guide. Test buffer mixtures as described in the text. Values preceded by $(<)$ or $(>)$ symbols indicate that that tolerable limit for the chemical is unknown but is, respectively, less than or greater than the amount shown. Blank spaces indicate data were unavailable.

b PCA, Perchloric acid; TCA, trichloroacetic acid; HEPES, $N$-2-hydroxyehtylpiperazine- $N$ '-2-ethanesulfonic acid; CHAPS, 3[(3-cholamidopropyl)dimethylammino]propanesulfonic acid; SDS, sodium dodecyl sulfate; (R), reduced; DMSO, dimethyl sulfoxide; EDTA ehtylenediamine-tetracetic acid.

${ }^{\mathrm{c}}$ Values indicate the concentration of the chemical in a $25 \mu \mathrm{L}$ sample.

${ }^{\mathrm{d}}$ Values indicate the final concentration of the chemical which does not produce an absorbance of 0.5 compared to an equivalent water blank.
}

Based on the various limits of detection for the different methods and the anticipated concentrations of proteins, standard curves should be prepared at the relevant solution conditions for the supernatant and coacervate samples. It is recommended that standard curves have at least five points and completely span the range of interest. Example standard curves corresponding to the supernatant and coacervate samples from Figure 4 are shown in Figure 5.

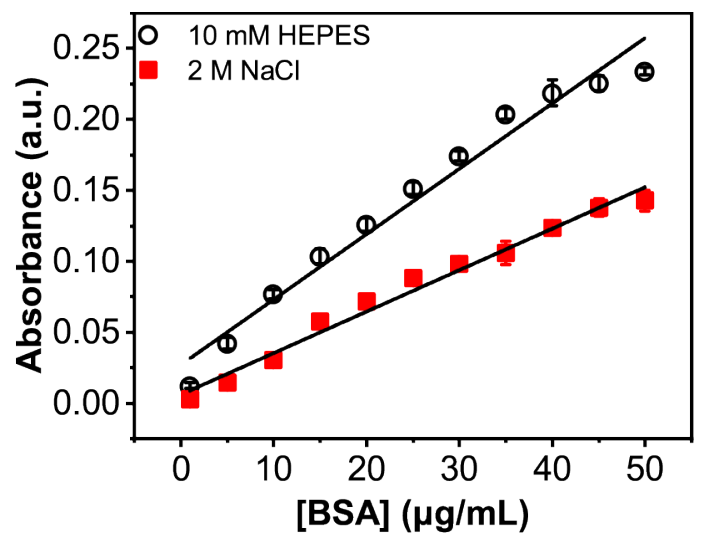

Figure 5: Standard curves for BSA for a Bradford assay under $10 \mathrm{mM}$ HEPES (black open circles) with a 1:1 dye to sample ratio and $2 \mathrm{M} \mathrm{NaCl}$ (red squares) with a 1:1 dye to sample ratio corresponding to the supernatant and coacervate phases. The black lines represent linear fits.

The best practice is to make new curves for every new cargo as they vary from protein to protein as seen comparing the curves from Figure 5 and Figure 6a. Additionally, varying the ratio of dye to sample can change the linear range of a protein. In the case of hen egg white lysozyme (HEWL), a ratio of two parts dye to one part sample is used to extend the range to $50 \mu \mathrm{g} / \mathrm{mL}$ (Figure 6a). If the same ratio was used as for BSA in Figure 5, the result is no longer linear (Figure 6b). The more dye added, the longer the linear range, this also allows for smaller sample volumes. 

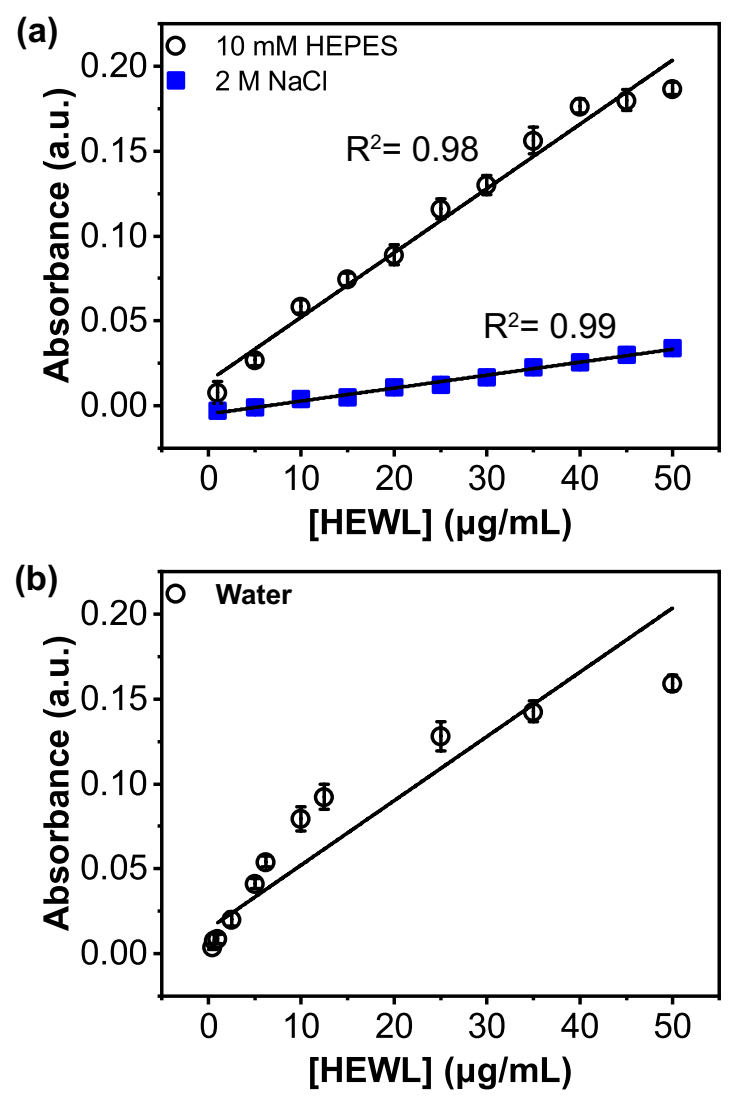

Figure 6: (a) Standard curves for HEWL for a Bradford assay under $10 \mathrm{mM}$ HEPES (black open circles) with a 2:1 dye to sample ratio and $2 \mathrm{M} \mathrm{NaCl}$ (blue squares) with a 1:1 dye to sample ratio corresponding to the supernatant and coacervate phases. (b) Standard curve for HEWL for a Bradford assay in water with a 1:1 dye to sample ratio, which shows a shorter linear range. The black lines represent linear fits to the data.

Table 5 shows an example recipe for a standard curve is outlined in Figures 5 and 6 to create a curve that would place the target concentration in the middle of the range at $\mathrm{pH} 7$ in $10 \mathrm{mM}$ HEPES. The goal is to create a linear curve over the desired range of concentrations using the Bradford. A curve for both the supernatant and coacervate phases is required. The supernatant will have the conditions set by sample preparation, while the coacervate phase will necessitate whatever conditions are used to dismantle the dense phase, i.e. $2 \mathrm{M} \mathrm{NaCl}$. To change Table 5 from the supernatant phase, take the total volume of buffer (HEPES) and water and add that amount of salt. 
Table 5: Standard curve example table for bovine serum albumin (BSA) or hen egg white lysozyme (HEWL) for the supernatant phase. Total solution volume is $120 \mu \mathrm{L}$ and can be used for a $96(120 \mu \mathrm{L} /$ well $)$ or 384-well plate $(35 \mu \mathrm{L} /$ well x3).

\begin{tabular}{c|c|c|c|c} 
Point & $\begin{array}{c}{[\text { Protein] }} \\
(\boldsymbol{\mu g} / \mathbf{m L})\end{array}$ & $\begin{array}{c}\mathbf{0 . 2} \mathbf{~ m g} / \mathbf{m L} \text { Protein } \\
(\boldsymbol{\mu} \mathbf{L})\end{array}$ & $\begin{array}{c}\mathbf{0 . 5} \text { M HEPES } \\
(\boldsymbol{\mu} \mathbf{L})\end{array}$ & $\begin{array}{c}\text { Water } \\
(\boldsymbol{\mu} \mathbf{L})\end{array}$ \\
\hline 1 & 1 & 0.6 & 2.4 & 117.0 \\
2 & 5 & 3.0 & 2.4 & 114.6 \\
3 & 10 & 6.0 & 2.4 & 111.6 \\
4 & 15 & 9.0 & 2.4 & 108.6 \\
5 & 20 & 12.0 & 2.4 & 105.6 \\
6 & 25 & 15.0 & 2.4 & 102.6 \\
7 & 30 & 18.0 & 2.4 & 99.6 \\
8 & 35 & 21.0 & 2.4 & 96.6 \\
9 & 40 & 24.0 & 2.4 & 93.6 \\
10 & 45 & 27.0 & 2.4 & 90.6 \\
11 & 50 & 30.0 & 2.4 & 87.6 \\
12 & 55 & 33.0 & 2.4 & 84.6 \\
13 & 60 & 36.0 & 2.4 & 81.6 \\
14 & 65 & 39.0 & 2.4 & 78.6 \\
15 & 70 & 42.0 & 2.4 & 75.6 \\
Blank & 0 & 0 & 2.4 & 117.6 \\
\hline
\end{tabular}

\section{Analysis and Statistics}

To facilitate statistical analysis, repeat samples and replicate experiments should be performed at least three times. A standard t-test or ANOVA may be used for comparison between assay results.

Moving beyond raw data to calculated values such as the concentration of protein in the coacervate phase, the encapsulation efficiency, partitioning, etc. require propagation of error. Furthermore, propagation of error should be considered when performing baseline subtraction. To obtain an equation for the propagated error use:

$$
\delta R=\sqrt{\left(\frac{\partial R}{\partial x} \delta x\right)^{2}+\left(\frac{\partial R}{\partial y} \delta y\right)^{2}+\cdots}
$$

where $R$ is the function whose error is being calculated and is a function of $x, y$, etc. The partial derivative of $R$ for a specific independent variable is multiplied by the error of that variable and the whole quantity squared. This is done for each variable for which $R$ is dependent and summed together. Finally, the square root of the summation is taken and that is the error for $R$. For example, the propagated error associated with performing a baseline subtraction between averaged values of the sample and baseline absorbance is:

$$
\delta A_{\text {avg }, b}=\sqrt{\left(\frac{\partial}{\partial b}\left(A_{\text {avg }}-b\right) \delta b\right)^{2}+\left(\frac{\partial}{\partial A_{\text {avg }}}\left(A_{\text {avg }}-b\right) \delta A_{\text {avg }}\right)^{2}}
$$

This type of calculation should be performed for each mathematical manipulation performed during data analysis. When using a standard curve, there is an associated error with the fit, which should be included using error propagation. This error can be determined by using Eq. 9 . 


\section{Summary}

Complex coacervates are a novel materials platform for the encapsulation and delivery of a wide range of materials, including proteins. We have discussed strategies for understanding the phase behavior associated with the coacervation of polymeric systems in the absence and presence of protein, along with methods for measuring the concentration of protein in coacervate samples. Here we have shown that it is possible to use established methods and adapt them to coacervate systems for protein analysis.

\section{Acknowledgements}

W.C.B.M. was supported by a Fellowship from the Soft Materials for Life Sciences National Research Traineeship Program \#NRT-1545399. We would also like to thank PPG for funding support of W.C.B.M. through a grant to the Department of Chemical Engineering at the University of Massachusetts Amherst. 


\section{References}

(1) Schmitt, C.; Turgeon, S. L. Protein/polysaccharide complexes and coacervates in food systems. Adv. Colloid Interface Sci. 2011, 167 (1-2), 63-70 DOI: 10.1016/j.cis.2010.10.001.

(2) Yeo, Y.; Bellas, E.; Firestone, W.; Langer, R.; Kohane, D. S. Complex Coacervates for Thermally Sensitive Controlled Release of Flavor Compounds. J. Agric. Food Chem. 2005, 53 (19), 7518-7525 DOI: 10.1021/jf0507947.

(3) Carvalho, I. T.; Estevinho, B. N.; Santos, L. Application of microencapsulated essential oils in cosmetic and personal healthcare products - a review. Int J Cosmet Sci 2016, 38 (2), 109-119 DOI: 10.1111/ics.12232.

(4) Martins, I. M.; Barreiro, M. F.; Coelho, M.; Rodrigues, A. E. Microencapsulation of essential oils with biodegradable polymeric carriers for cosmetic applications. Chemical Engineering Journal 2014, 245 (C), 191-200 DOI: 10.1016/j.cej.2014.02.024.

(5) Kuo, C.-H.; Leon, L.; Chung, E. J.; Huang, R.-T.; Sontag, T. J.; Reardon, C. A.; Getz, G. S.; Tirrell, M.; Fang, Y. Inhibition of atherosclerosis-promoting microRNAs via targeted polyelectrolyte complex micelles. J. Mater. Chem. B 2014, 2, 8142-8153 DOI: 10.1039/C4TB00977K.

(6) Water, J. J.; Schack, M. M.; Velazquez-Campoy, A.; Maltesen, M. J.; van de Weert, M.; Jorgensen, L. Complex coacervates of hyaluronic acid and lysozyme: Effect on protein structure and physical stability. 2014, 88 (2), 325-331 DOI: 10.1016/j.ejpb.2014.09.001.

(7) Black, K. A.; Priftis, D.; Perry, S. L.; Yip, J.; Byun, W. Y.; Tirrell, M. Protein encapsulation via polypeptide complex coacervation. ACS Macro Lett. 2014, 3 (10), 1088-1091 DOI: 10.1021/mz500529v.

(8) Kishimura, A.; Koide, A.; Osada, K.; Yamasaki, Y.; Kataoka, K. Encapsulation of Myoglobin in PEGylated Polyion Complex Vesicles Made from a Pair of Oppositely Charged Block Ionomers: A Physiologically Available Oxygen Carrier. Angew. Chem. Int. Ed. 2007, 46 (32), 6085-6088 DOI: 10.1002/anie.200701776.

(9) Vehlow, D.; Schmidt, R.; Gebert, A.; Siebert, M.; Lips, K.; Müller, M. Polyelectrolyte complex based interfacial drug delivery system with controlled loading and improved release performance for bone therapeutics. Nanomaterials 2016, 6 (3), 53-21 DOI: 10.3390/nano6030053.

(10) Lindhoud, S.; Voorhaar, L.; de Vries, R.; Schweins, R.; Cohen Stuart, M. A.; Norde, W. Salt-Induced Disintegration of Lysozyme-Containing Polyelectrolyte Complex Micelles. Langmuir 2009, 25 (19), 11425-11430 DOI: 10.1021/la901591p.

(11) Lindhoud, S.; de Vries, R.; Schweins, R.; Cohen Stuart, M. A.; Norde, W. Salt-induced release of lipase from polyelectrolyte complex micelles. Soft Matter 2009, 5 (1), 242250 DOI: 10.1039/B811640G.

(12) Lim, Z. W.; Ping, Y.; Miserez, A. Glucose-Responsive Peptide Coacervates with High Encapsulation Efficiency for Controlled Release of Insulin. Bioconjugate Chem. 2018, 29 (7), 2176-2180 DOI: 10.1021/acs.bioconjchem.8b00369.

(13) Nishida, K.; Tamura, A.; Yui, N. pH-Responsive Coacervate Droplets Formed from Acid-Labile Methylated Polyrotaxanes as an Injectable Protein Carrier.

Biomacromolecules 2018, 19 (6), 2238-2247 DOI: 10.1021/acs.biomac.8b00301.

(14) Johnson, N. R.; Wang, Y. Coacervate delivery systems for proteins and small molecule drugs. Expert Opin. Drug Delivery 2014, 11 (12), 1829-1832 DOI:

10.1517/17425247.2014.941355. 
(15) Johnson, N. R.; Wang, Y. Controlled delivery of heparin-binding EGF-like growth factor yields fast and comprehensive wound healing. J. Controlled Release 2013, 166 (2), 124-129 DOI: 10.1016/j.jconrel.2012.11.004.

(16) Chapeau, A.-L.; Bertrand, N.; Briard-Bion, V.; Hamon, P.; Poncelet, D.; Bouhallab, S. Coacervates of whey proteins to protect and improve the oral delivery of a bioactive molecule. J Funct Foods 2017, 38, 197-204 DOI: 10.1016/j.jff.2017.09.009.

(17) Bourganis, V.; Karamanidou, T.; Kammona, O.; Kiparissides, C. Polyelectrolyte complexes as prospective carriers for the oral delivery of protein therapeutics. Eur $J$ Pharm Biopharm 2017, 111, 44-60 DOI: 10.1016/j.ejpb.2016.11.005.

(18) Obermeyer, A. C.; Mills, C. E.; Dong, X.-H.; Flores, R. J.; Olsen, B. D. Complex coacervation of supercharged proteins with polyelectrolytes. Soft Matter 2016, 12, 3570-3581 DOI: 10.1039/C6SM00002A.

(19) Cummings, C. S.; Obermeyer, A. C. Phase Separation Behavior of Supercharged Proteins and Polyelectrolytes. Biochemistry 2018, 57 (3), 314-323 DOI: 10.1021/acs.biochem.7b00990.

(20) Perry, S.; Li, Y.; Priftis, D.; Leon, L.; Tirrell, M. The effect of salt on the complex coacervation of vinyl polyelectrolytes. Polymers 2014, 6 (6), 1756-1772 DOI: 10.3390/polym6061756.

(21) Priftis, D.; Tirrell, M. Phase behaviour and complex coacervation of aqueous polypeptide solutions. Soft Matter 2012, 8 (36), 9396-9405 DOI: 10.1039/C2SM25604E.

(22) Priftis, D.; Megley, K.; Laugel, N.; Tirrell, M. Complex coacervation of poly(ethyleneimine)/polypeptide aqueous solutions: thermodynamic and rheological characterization. J. Colloid Interface Sci. 2013, 398, 39-50 DOI: 10.1016/j.jcis.2013.01.055.

(23) Chollakup, R.; Smitthipong, W.; Eisenbach, C. D.; Tirrell, M. Phase Behavior and Coacervation of Aqueous Poly(acrylic acid)-Poly(allylamine) Solutions.

Macromolecules 2010, 43 (5), 2518-2528 DOI: 10.1021/ma902144k.

(24) Chollakup, R.; Beck, J. B.; Dirnberger, K.; Tirrell, M.; Eisenbach, C. D. Polyelectrolyte molecular weight and salt effects on the phase behavior and coacervation of aqueous solutions of poly(acrylic acid) sodium salt and poly(allylamine) hydrochloride.

Macromolecules 2013, 46 (6), 2376-2390 DOI: 10.1021/ma202172q.

(25) Comert, F.; Dubin, P. L. Liquid-liquid and liquid-solid phase separation in proteinpolyelectrolyte systems. Adv. Colloid Interface Sci. 2017, 239, 213-217 DOI: 10.1016/j.cis.2016.08.005.

(26) Kaibara, K.; Okazaki, T.; Bohidar, H. B.; Dubin, P. L. pH-Induced Coacervation in Complexes of Bovine Serum Albumin and Cationic Polyelectrolytes.

Biomacromolecules 2000, 1 (1), 100-107 DOI: 10.1021/bm990006k.

(27) Yan, Y.; Kizilay, E.; Seeman, D.; Flanagan, S.; Dubin, P. L.; Bovetto, L.; Donato, L.; Schmitt, C. Heteroprotein complex coacervation: bovine $\beta$-lactoglobulin and lactoferrin. Langmuir 2013, 29 (50), 15614-15623 DOI: 10.1021/la4027464.

(28) Madinya, J. J.; Chang, L.-W.; Perry, S. L.; Sing, C. E. Sequence-dependent selfcoacervation in high charge-density polyampholytes. Mol. Syst. Des. Eng. 2019, Accepted DOI: 10.1039/C9ME00074G.

(29) Li, L.; Srivastava, S.; Andreev, M.; Marciel, A. B.; de Pablo, J. J.; Tirrell, M. V. Phase Behavior and Salt Partitioning in Polyelectrolyte Complex Coacervates.

Macromolecules 2018, 51 (8), 2988-2995 DOI: 10.1021/acs.macromol.8b00238.

(30) Chang, L.-W.; Lytle, T. K.; Radhakrishna, M.; Madinya, J. J.; Vélez, J.; Sing, C. E.; 
Perry, S. L. Sequence and entropy-based control of complex coacervates. Nat Commun 2017, 8, 1273 DOI: 10.1038/s41467-017-01249-1.

(31) Xu, Y.; Liu, M.; Faisal, M.; Si, Y.; Guo, Y. Selective protein complexation and coacervation by polyelectrolytes. Adv. Colloid Interface Sci. 2017, 239, 158-167 DOI: 10.1016/j.cis.2016.06.004.

(32) Comert, F.; Malanowski, A. J.; Azarikia, F.; Dubin, P. L. Coacervation and precipitation in polysaccharide-protein systems. Soft Matter 2016, 12, 4154-4161 DOI: 10.1039/C6SM00044D.

(33) Xu, Y.; Mazzawi, M.; Chen, K.; Sun, L.; Dubin, P. L. Protein Purification by Polyelectrolyte Coacervation: Influence of Protein Charge Anisotropy on Selectivity. Biomacromolecules 2011, 12 (5), 1512-1522 DOI: 10.1021/bm101465y.

(34) Cooper, C. L.; Dubin, P. L.; Kayitmazer, A. B.; Turksen, S. Polyelectrolyte-protein complexes. Curr. Opin. Colloid Interface Sci. 2005, 10 (1-2), 52-78 DOI: 10.1016/j.cocis.2005.05.007.

(35) Blocher McTigue, W. C.; Perry, S. L. Design Rules for Encapsulating Proteins into Complex Coacervates. Soft Matter 2019, 15, 3089-3103 DOI: 10.1039/C9SM00372J.

(36) Lindhoud, S.; Claessens, M. M. A. E. Accumulation of small protein molecules in a macroscopic complex coacervate. Soft Matter 2016, 12 (2), 408-413 DOI: 10.1039/C5SM02386F.

(37) Lindhoud, S.; Norde, W.; Cohen Stuart, M. A. Reversibility and Relaxation Behavior of Polyelectrolyte Complex Micelle Formation. J. Phys. Chem. B 2009, 113 (16), 54315439 DOI: $10.1021 / \mathrm{jp} 809489 f$.

(38) Olson, B. J. S. C.; Markwell, J. Assays for determination of protein concentration.; John Wiley \& Sons, Inc.: Hoboken, NJ, USA, 2007; Vol. Chapter 3, pp Unit3.4-3.4.29.

(39) Bio-Rad Laboratories. Quick Start ${ }^{T M}$ Bradford Protein Assay; pp 1-36.

(40) Stoscheck, C. M. Quantitation of Protein. Methods in Enzymology 1990, 182, 50-68.

(41) Simonian, M. H. Spectrophotometric Determination of Protein Concentration. Curr Protoc Cell Biol 2002, 15 (1), A.3B.1-A.3B.7 DOI: 10.1002/0471143030.cba03bs15.

(42) Thermo Scientific. Protein A280; 2010; pp 1-32.

(43) Gasteiger, E.; Hoogland, C.; Gattiker, A.; Duvaud, S.; Wilkins, M. R.; Appel, R. D.; Bairoch, A. The Proteomics Protocols Handbook; Walker, J. M., Ed.; Humana Press, 2005; pp 571-607. 